\title{
Manipulation and separation of oil droplets by using asymmetric nano-orifice induced DC dielectrophoretic method
}

\author{
Kai Zhao, Dongqing Li* \\ Department of Mechanical and Mechatronics Engineering \\ University of Waterloo \\ Waterloo, Ontario, Canada N2L 3G1
}

*Corresponding author, Email: dongqing@uwaterloo.ca 


\section{Abstract \\ Hypothesis}

Emulsion droplets are versatile tools in chemical and biological fields and the separation of emulsion droplets is one of the most crucial steps in research and industry.

\section{Experiments}

This work developed a nano-orifice based DC dielectrophoretic(DEP) system for the sorting of oil-in-water emulsion droplets by size and content in a microchannel. Then, the size-dependent separation of oil droplets was conducted and verified by the prediction of theoretical simulation. In addition, by selecting the suspending solution with a specific electrical conductivity, the oil droplets with similar sizes but different contents were separated based on their opposite DEP behaviors.

\section{Findings}

The size-dependent separation of smaller silicone oil droplets (7.5 and $11 \mu \mathrm{m}$ in diameter) with a high separation resolution, i.e., size difference of only $3.5 \mu \mathrm{m}$ was achieved and showed good agreement with the simulation results. The positive and negative DEP behaviors of the droplets varying with the electrical conductivity of the suspending solution were discussed and different types of droplets of similar sizes but different contents were separated. This paper presents the DCDEP method based sorting of emulsion droplets by size and content in nanofluidic chip for the first time, providing a platform to manipulate individual emulsion droplet.

\section{Keywords:}

Electrokinetics, Nano-orifice, Dielectrophoretic separation, Oil-in-water emulsion droplets 


\section{Introduction}

Emulsions with liquid droplets dispersing in another insoluble liquid have been versatile tools in chemical, biological, as well as medical fields. The emulsion droplets is applicable for the fabrication of solid particles [1-3], encapsulation, and screening of cells and DNA [4-8], as well as drug delivery and screening [9]. As the droplets have good compatibility with many chemical and biological reagents, the droplet-based microfluidic technology enables the field of single-cell biology [10,11], and the droplets can function as individual microreactors and serve as carriers with a variety of kinds of biochemical reactions [12-14]. Due to the dimensional scaling benefits, the reactions of fluids in the droplet is controllable and rapid, resulting in reduced reagent consumption and high manipulation efficiencies [15]. Generally, the droplet microfluidic involves the production and manipulation of the individual droplet in the microchannels [16], and it is crucial to fabricate the droplets in a uniform structure during that process. In spite of the highlydemanded applications of the droplets, the droplet generation techniques are limited. Normally, the droplets are manufactured by stirring the mixture with large-scale instruments [17]. It is usually difficult to produce the droplets with the precise size and typically the droplets have wide size distributions [18]. Alternatively, the microfluidics has become a prevalent technique to produce highly monodisperse emulsion droplets [19]. Depending on the channel geometry in the microfluidic device, the T-junction [20-23] and flow-focusing [24-27] configurations are introduced to form droplets with controllable sizes. Recent improvements in microfluidic emulsion technology enable the generation of uniform emulsion droplets in a small volume by electrical control, such as dielectrophoretic [28-30] and electrowetting on dielectric methods [31-33]. These fabrication methods can generate large numbers of droplets with a diameter which ranges from nanometers to micrometers [34]. However, it still shows a variation of $3-10 \%$ in size of the emulsion droplets produced by these methods [35]. Moreover, the production of the daughter droplets together with the mother droplets is undesired and then the quality of the droplets becomes hard to control [36]. Thus, it is essential to develop a tool for sorting emulsion droplets by size and the droplets with well-defined size and uniform morphology can be purified for consecutive applications.

Recently, the manipulation of emulsion droplets attracts considerable interest and the development of the related microfluidic technology provides various approaches for sorting emulsion droplets in the microchannel, which include gravity and microchannel geometry effects [37-39], 
mechanical valves [40-42], electrowetting-on-dielectric (EWOD) [43], dielectrophoresis (DEP) [44-48], etc. As the sedimentation velocity of the smaller droplets is lower than that of the larger droplets, Huh et al. [39] designed the gravity-driven sized-based separation system in the microchannel to separate the droplets with different diameter by employing the hydrodynamic sorting amplification. By using the asymmetrical widening design for the separation microchannel, the sorting effect is enhanced. However, this method has limited sensitivity for droplets of similar sizes. The droplets can also be separated mechanically by using valves which are assembled in the microfluidic devices. In the design created by Abate et al. [40], the fluid flow is manipulated by the single-layer membrane valve which is fabricated in the microchannel, enabling the separation of droplets into different outlets with high speed at a frequency of hundreds of hertz. However, in order to sort droplets effectively by this method, precise control over the flow field inside the microchannel is necessary. The EWOD method is also utilized for sorting droplets. Cho et al. [43] conducted the separation of droplets and its contents in the EWOD-based microfluidic chip by adjusting the interfacial energy of the droplets and the solid surface with an array of microelectrodes, resulting in the droplets movement. Two different kinds of particles were first devided by electrophoresis into two areas of a master droplet and then splitted the droplet in half by EWOD actuation. Then the two daughter droplets with different contents can be separated. Even though the EWOD technology enables to achieve separation inside the droplets, its throughput is very limited in comparison with the high-speed production and performance of the flow-focused based droplet systems.

In addition to the above-mentioned separation methods, dielectrophoresis proves to be an effective strategy for continuously sorting particles and droplets by size and its content in microfluidic systems [44-46,48-53] due to its advantages of low sample consumption, label-free separation, and fast response. Due to the dielectric proerty differences of the droplets and the suspending solution in the electrical field gradient, the DEP force is induced and drives the droplets to move. The non-uniform electric field can be induced by employing the electric potential to an array of asymmetrical microelectrodes embedded inside the microchannel or via the external electrodes across the varying shape of the microchannel with insulator-based constricted structures [54]. Since the magnitude of the DEP force varies with the size of the emulsion droplets, the sizedependent separation of the emulsion droplets is straightforward. While, to sort the droplets of similar sizes but different contents, it always requires discrete processes [53]. As different particles 
or droplets show distinct dielectric property, the alternating current (AC) DEP is applied to identify and separate the particles or droplets by adjusting the applied frequency [55]. In the design demonstrated by Ahn et al. [44], the DEP effect was first introduced for high-throughput droplets sorting. In order to drive the droplets into designated channel branches, indium tin oxide (ITO) microelectrodes with specific location and structures were placed underneath the PDMS microchannels to provide the maximized field gradient and force, leading to a sorting rate of 1.6 kHz. Guo et al. [48] introduced a droplet electric sorting microfluidic chip for cell separation. By optimizing the electrodes and the electric square pulse, single or multiple mammalian cells encapsulated alginate droplet can be isolated, respectively, which provides a method for the operation and analysis of single cell. The microfluidic platform combining with optically induced DEP was introduced to manipulate and separate the droplets [45,56-58]. By introducing optical images onto the photoconductive materials on the opposite sides of the microchannel, the virtual electrodes were produced where generates the electric field gradient and hence the DEP force. Instead of utilizing fixed microelectrodes, the moving electrodes images provided a convenient method and enabled the programmed movement of the individual droplet by adjusting the laser beam. Generally, the emulsion droplets can be manipulated in microfluidic chips by utilizing the conventional DEP effects. However, the implementation of the microelectrodes in the microfluidic chips and the fabrication processes are difficult and expensive. Furthermore, the traditional dielectrophoretic methods cannot achieve the sorting of emulsion droplets with both high separation resolution and throughput. The problems discussed above can be overcome by a newly developed DEP method using asymmetric orifices [49-51]. As discussed elsewhere [49-51], the electrical field gradient can be induced by employing the DC electric voltages through a group of asymmetric orifices through the external electrodes. By using a pair of asymmetric orifices with large width ratio, a stronger electric field gradient and hence larger DEP force can be produced with a strong electric field strength applied through the microchannel, resulting in a high separation resolution. Furthermore, in this design, the production of the electric field gradient will not change the cross-section of the microchannel and has no influence on the particle or droplet transportation. By using the pressure-driven flow to drive the particles and droplets, the throughput can be dramatically increased in comparison with other microfluidic DEP methods.

It is well-known that manipulation and separation of micro emulsion oil droplets are critical aspects in colloid and interface science. In this paper, the manipulation and sorting of the oil-in- 
water emulsion droplets was conducted inside the microchannel by the asymmetric orifice based DC-DEP. The fabrication of the nano-orifice based microfluidic chip and the distribution of the electric fields near the small orifice were presented. Then, the size-dependent separation of emulsion droplets was conducted, and the prediction of theoretical simulation was compared with the experimental results. In addition, selecting the suspending solution with a specific electrical conductivity, the oil droplets with similar sizes but different contents were separated based on their opposite DEP behaviors. This paper, for the first time to our knowledge, presents the sizedependent and content-denpendent separation of emulsion oil droplets under a pressure-driven flow in the nano-orifice based nanofluidic channel by using DC-DEP method, broadening the applications of the DC-DEP method in colloid and interface science and in industry.

\section{Materials and Methods}

\subsection{Dielectrophoretic separation}

Dielectrophoresis is the motion of a polarized particle/droplet in an aqueous solution with the presence of the non-uniformity of an electric field. The DEP force exerting on the particle/droplet is generally expressed as [59]

$$
\mathrm{F}_{\mathrm{DEP}}=2 \pi \varepsilon_{m} a^{3} \operatorname{Re}\left(f_{\mathrm{CM}}\right)\left(\nabla|E|^{2}\right)
$$

where $a$ and $\varepsilon_{m}$ represents the droplets radius and the electrical permittivity of the surrounding medium, the electrical field squared gradient is demonstrated by $\nabla|E|^{2}$, and the real part of the Clausius-Mossotti (CM) factor, i.e., $\operatorname{Re}\left(f_{\mathrm{CM}}\right)$ is expressed as

$$
f_{\mathrm{CM}}=\left(\frac{\varepsilon_{p}^{*}-\varepsilon_{m}^{*}}{\varepsilon_{p}^{*}+2 \varepsilon_{m}^{*}}\right)
$$

where droplet and the solution is illustrated by the subscripts $p$ and $m$, respectively, and the complex permittivity, i.e., $\varepsilon^{*}$ is described as

$$
\varepsilon^{*}=\varepsilon-(j \sigma / \omega)
$$

where the permittivity and electric conductivity of the droplet and the surrounding medium are represented by $\varepsilon$ and $\sigma$, respectively, the angular frequency of the electric field is represented by $\omega$, and $j=\sqrt{-1}$. The $f_{\mathrm{CM}}$ demonstrates the relative polarizabilities between the droplets and the surrounding medium. A positive value of the $f_{\mathrm{CM}}$ indicates that the particles/droplets are more polarizable than the suspending solution, and they will be attracted by the positive DEP (p-DEP) forces and flow towards the maximum electric field. While a negative value of $f_{\mathrm{CM}}$ indicates that 
the particles/droplets are less polarizable than the medium which will undergo the negative-DEP (n-DEP) effects and be pushed away from the maximum electric field. When employing the DC electric fields, the $f_{\mathrm{CM}}$ becomes solely dependent on the electrical conductivity of the droplet $\sigma_{p}$ and the surrounding medium $\sigma_{m}$ which is expressed as [60]

$$
f_{C M}=\left(\frac{\sigma_{p}-\sigma_{m}}{\sigma_{p}+2 \sigma_{m}}\right)
$$

It can be inferred from Eq (1) that the magnitude of the dielectrophoretic forces will increase with the electric field gradient $\left(\nabla|E|^{2}\right)$ and the radius cubic of the droplet $\left(a^{3}\right)$, while the sign of $f_{\mathrm{CM}}$ determines the direction of the DEP effects. Since the trajectory shifts are proportional to the DEP forces acting on the droplets, the droplets with different radius can be separated and flow into individual streams. In order to achieve a high separation resolution, a strong electric field gradient is necessary. In this work, a pair of asymmetric orifices with a sufficiently large width ratio is used to induce a strong electric field gradient, illustrated in Figure 1. As the distance between the two external electrodes is relatively small (essentially over the width of the microchannel), a low electrical potential difference applied through the orifices across the microchannel is enough to generate the strong electric field gradient.

In addition, since the direction of the dielectrophoretic forces, i.e., p-DEP and n-DEP, is determined by the sign of $f_{\mathrm{CM}}$, two different kinds of particles/droplets may have opposite DEP behaviors and hence can be separated. It can be inferred from Eq (4) that, for the droplets whose electrical conductivity is smaller than that of the suspending solution, i.e., $f_{\mathrm{CM}}<0$, they will be repelled away by the n-DEP behaviors from the electric field maximum. While for the droplets that has a larger electrical conductivity than the medium, i.e., $f_{\mathrm{CM}}>$, they will be attracted by $\mathrm{p}$-DEP forces towards the electric field maximum. Therefore, by using the DC-DEP device as shown in Figure 1 , not only the separation of droplets with different sizes can be achieved, but also the separation of droplets with similar sizes but different contents (and hence different electrical conductivity) can also be accomplished by selecting a suspending solution with an electrical conductivity, $\sigma_{p 1}<$ $\sigma_{m}<\sigma_{p 2}$

\subsection{Fabrication of nanofluidic chips}

The nanofluidic chips are composed of a polydimethylsiloxane (PDMS) microchannel in the top and a single nanochannel in the bottom. The microchannel mold is designed by the AutoCAD 
software and microchannel in the top is produced by peeling off the PDMS-curing agent mixture from the silicon wafer master by the standard photolithography technology (MicroChem, SU-8 photoresist). The bottom PDMS layer with a single nanochannel is replicated from the nanochannel mold which is fabricated by the following steps [49,51,61]. Firstly, a nano-crack with controlled size is created on the polystyrene surface (Petri dish surface, VWR) by using a solvent-based method. Then, the nano-crack is transferred onto an SU-8 photoresist layer to work as a positive nanochannel mold by the soft photolithography method. To avoid the collapse of the nanochannel roofs after bonding, the hard PDMS [62] with a higher Young's modulus is spin-coated on the nanochannel mold and microchannel mold followed by heating in the oven at $70{ }^{\circ} \mathrm{C}$ for 1 hour. Then, a regular PDMS layer is cast onto the hard PDMS followed by heating in the oven at $80{ }^{\circ} \mathrm{C}$ for another 2 hours. After peeling off from the channel molds, the top layer (PDMS microchannel) and the bottom layer (PDMS nanochannel) are treated by plasma ((Harrick plasma ${ }^{\circledR}$, PDC-32G) and the nanofluidic chips is eventually obtained by bonding these two layers together with a homemade alignment system under the microscope.

As shown in Figure 1(A), the structure of this nanofluidic chip includes a main channel with the sample inlet channel (A) and pressure-driven fluid inlet channel (B), the outlet chennels (E and F), as well as two wells (C and D) where inserting the external electrodes. In order to induce the electric field gradient and hence the dielectrophoretic forces, the micron and nano orifices are fabricated on the opposite sides of the mainchannel walls. As shown in Figure (A), when the mixture of the emulsion droplets comes into the horizontal main channel through the sample inlet channel A, they are forced to flow closely to the side wall of the small orifice by the hydrodynamically dominant flow from the sheath fluid inlet channel B. Meanwhile, a DC electrical potential difference is applied through the pair of the orifices by the external electrodes, where the stream line of the electric field across the channel between the $\mathrm{C}$ and $\mathrm{D}$ is limited by the opening sizes of the asymmetric orifices. To ensure the production of a large electric field gradient near the nano-orifice, the asymmetric orifices have a large width ratio. Then, the droplets will experience the DEP effects when moving through the small orifice where the strongest gradient of the electric field exists.

In this chip, the width of the branch channels B, E and F and the main channel are $80 \mu \mathrm{m}$, and the width of the branch channel A is $20 \mu \mathrm{m}$. The depth of the whole channel is $25 \mu \mathrm{m}$. In this study, 
the local electric field is produced by the platinum electrodes inserted in the reservoirs C and D where a DC electric potential is applied, and the distance between these two external electrodes is approximately $0.6 \mathrm{~cm}$. In order to induce strong electric field gradient, a single nanochannel of $860 \mathrm{~nm}$ in width and $290 \mathrm{~nm}$ in depth is utilized as the nano-orifice. The length of the nano-orifice is always $15 \mu \mathrm{m}$ and the large orifice has a width of $125 \mu \mathrm{m}$ for all chips.

An example of the PDMS nanofluidic chip after bonding is shown in Figure 2(A) and a zoom-in view of the asymmetric orifices area captured by using an optical microscope (Nikon, TE-2000) is shown in Figure 2(B). The nanochannel used in this work is characterized by using an AFM

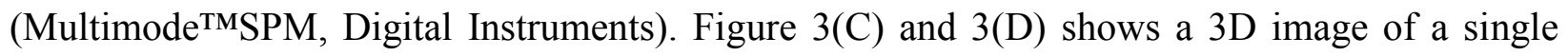
PDMS nanochannel which is measured by the AFM and has a width of $860 \mathrm{~nm}$ and a depth of 290 $\mathrm{nm}$.

\subsection{Preparation of oil-in-water emulsion}

The emulsion droplets were fabricated by mixing the silicone oil (XIAMETER PMS-200) or the silicone oil-based Carbon and Silver Conductive Grease (MG Chemicals) with water by utilizing the nonionic surfactant Tween 20 (Sigma Aldrich) as the stabilizing agent. After introducing $5 \mathrm{~mL}$ DI water, $1 \mathrm{~mL}$ silicone oil with $100 \mu \mathrm{L}$ surfactant solution or $0.05 \mathrm{~g}$ conductive grease with 50 $\mu \mathrm{L}$ surfactant solution into a glass bottle $(15 \mathrm{~mL}$ in volume), the emulsion droplets were generated by stirring the mixture with an ultrasonic clearner at the frequency of $42 \mathrm{kHz}$ for 2 minutes (Cody Digital Ultrasonic Cleaner) and a vortex mixer (VWR Scientific) at the speed of $3200 \mathrm{rpm}$ for 1 minute.

\subsection{Experimental system setup}

In this study, the two pairs of silicone oil droplets, i.e., $9 \mu \mathrm{m}$ and $14.5 \mu \mathrm{m}$, and $7.5 \mu \mathrm{m}$ and $11 \mu \mathrm{m}$, and three pairs of oil mixture droplets, i.e., droplets of silicone oil and carbon grease with a diameter of approximately $7.5 \mu \mathrm{m}$, droplets of silicone oil and carbon-plus-silver grease with a diameter of approximately $7 \mu \mathrm{m}$, as well as droplets of carbon grease and carbon-plus-silver grease with a diameter of approximately $7.5 \mu \mathrm{m}$ were fabricated. A solution of $0.4 \mathrm{mM} \mathrm{K}_{2} \mathrm{HPO}_{4}$ with a $\mathrm{pH}$ of 7 and an electrical conductivity of $0.01 \mathrm{~S} / \mathrm{m}$ was employed to separate the $7.5 \mu \mathrm{m}$ droplets of carbon grease and carbon-plus-silver grease. The deionized water with a $\mathrm{pH} 7$ and an electrical conductivity of $5.5 \times 10^{-6} \mathrm{~S} / \mathrm{m}$ was used for the rest groups of separation.

In the experiments, the suspending medium was added into the chip, at room temperature $25 \pm$ 
$1^{\circ} \mathrm{C}$. Then, $10 \mu \mathrm{L}$ of the emulsion droplets was introduced into the sample inlet well $\mathrm{A}, 7.5 \mu \mathrm{L}$ of the suspending solution was introduced into the sheath fluid inlet well B, $2.5 \mu \mathrm{L}$ of the suspending medium was injected into the reservoirs $\mathrm{C}$ and $\mathrm{D}$ to submerge the electrodes. Due to the limitation of the width of the microchannel, only the emulsion droplets with a radius smaller than $10 \mu \mathrm{m}$ were allowed to move into the horizontal channel. Then, the mixture of the droplets was pushed by the focusing liquid from branch channel B to move along the upper sidewall of the horizontal channel and experience the strong DEP effects near the nano-orifice. In this work, the pressuredriven flow was employed to drive the droplets and the liquid flow, which is controlled by the liquid height differences between the inlet and outlet wells. After the microchannel is wet by the suspending solution, $10 \mu \mathrm{L}$ of the droplet samples are introduced into the reservior A and no solution is introduced into outlet well $\mathrm{E}$ and $\mathrm{F}$. By adjusting the volume of the inlet sample solution, different flow rates can be obtained. The volume difference is used to adjust the liquid height differences between the inlet and outlet wells and hence the pressure difference. After placing the platinum electrodes into the reservoirs $\mathrm{C}$ and $\mathrm{D}$, the electric field was applied with DC power supplier (HVS448 High Voltage Sequencer, LabSmith). By adjusting the output electric potentials to the electrodes by a custom-made voltage adjuster, the strength of the electric field became controllable. As shown in Figure 3(B), the $\nabla|E|_{\max }^{2}$ calculated by COMSOL is in the order of $\sim 10^{21} \mathrm{~V}^{2} / \mathrm{m}^{3}$ with $320 \mathrm{~V}$ applied via a pair of electrodes over $0.15 \mathrm{~cm}$ between the reservoirs $\mathrm{C}$ and D. An inverted optical microscope (Nikon, TE-2000) was utilized to visualize the movements of the oil droplets in the horizontal channel and a charge coupled device CCD camera (QImaging) was employed to record the images at 25 frames per second.

\section{Results and discussion}

\subsection{Simulation of electric field and the oil droplet trajectory}

As shown in Figure 3, an example of the trajectories of the $7.5 \mu \mathrm{m}$ and $11 \mu \mathrm{m}$ silicone oil droplets and the distribution of the electric field near the asymmetric orifices are demonstrated. These are calculated by using COMSOL 4.3b. In this design, the non-uniformity of the electric field is produced by applying the $\mathrm{DC}$ electric potential through the nano and micron orifices by the external electrodes. The greatest non-uniformity of the electric field is produced neear the nanoorifice. As shown in Figure 3 (A), the oil droplets are loaded into the horizontal channel and forced by the dominant sheath flow from branch channel B to move close to the upper sidewall and pass over the nano-orifice where the greatest electric field gradient exists. It can be inferred from Figure 
3(B), the oil droplets are driven into individual trajectories after experiencing the dielectrophoretic effects. Since the locally applied electric potential is low and the nano-orifice has a width of 860 $\mathrm{nm}$, the liquid flow from reservoir $\mathrm{C}$ to the main channel can be neglected. As the magnitude of the DEP force is dependent on the radius cubic of the droplet, the larger $11 \mu \mathrm{m}$ oil droplets experience stronger n-DEP force, leading to a sufficiently large trajectory shift, and are pushed away into the outlet channel F. While, the DEP effects on the smaller $7.5 \mu \mathrm{m}$ oil droplets are relatively weaker and they flow into outlet channel E. If the mixed emulsion droplets have similar sizes but different contents, the droplets with stronger polarizability than that of the suspending solution will be attracted by the p-DEP effects towards the nano-orifice and flow into the outlet channel E. While the droplets, whose polarizability is weaker compared with that of the medium, will undergo n-DEP and be pushed towards the large orifice where has weak electric field, and then move into the outlet reservior F. Therefore, the size-dependent separation of the oil droplets and the separation of the oil droplets by its content can be achieved by using the nano-orifice based DC-DEP device.

\subsection{Size-dependent separation of oil droplets}

The separation of $7.5 \mu \mathrm{m}$ and $11 \mu \mathrm{m}$ silicone oil droplets, and $9 \mu \mathrm{m}$ and $14.5 \mu \mathrm{m}$ silicone oil droplets were conducted under DC-DEP effects by applying an electric potential to electrode C of $320 \mathrm{~V}$ and $240 \mathrm{~V}$, respectively, and the electrode $\mathrm{D}$ is grounded. The DI water with a $\mathrm{pH}$ value of 7 and an electric conductivity of $5.5 \times 10^{-6} \mathrm{~S} / \mathrm{m}$ was utilized as the suspending medium, and hence the silicone oil droplets will experience the n-DEP forces. In the experiments, the nano-orifice has a width of $860 \mathrm{~nm}$ and a length of $15 \mu \mathrm{m}$, and the large orifice has a width of $125 \mu \mathrm{m}$ in all nanofluidic chips. An example of the size-dependent separation of oil droplets is illustrated in Figure 4, where the trajectories of the oil droplets are obtained by superimposing a series of sequentially recorded images of the moving droplets.

As discussed about Eq (1), the dielectrophoretic forces and hence the lateral shifts of the oil droplets are dependent upon the electric field gradient and the diameter of droplets. Thus, as shown in Figure 1(A), the oil droplets of different radii are sorted into individual trajectory and separated when moving closely to the small orifice in the mainchannel. An examples of the size-dependent separation of the emulsion droplets are illustrated in Figure 4. The mixture of oil droplets flows into the channel A and they are pushed by the sheath fluid from branch channel B to move along 
the upper channel wall. When the silicone oil droplets move through the region of the electric field gradient, the n-DEP forces take effects, leading to the lateral shifts of their trajectories, and they are thus moved into different streams. By controlling the custom-made voltage adjuster, the smaller and larger oil droplets flow into the outlet channel $\mathrm{E}$ and outlet channel F, respectively. Under $\phi_{C}=240 \mathrm{~V}$, the larger oil droplets with $\mathrm{d}=14.5 \mu \mathrm{m}$ experience stronger DEP forces and flow into the outlet $F$, while the oil droplets with smaller diameter of $d=9 \mu \mathrm{m}$ experience weaker DEP effects and flow into the outlet E (Figure 4(A)). The experimental result (Figure 4(A)) shows good agreement with the simulation trajectories of the $9 \mu \mathrm{m}$ and $14.5 \mu \mathrm{m}$ diameter silicone oil droplets (Figure 3(A)). Furthermore, based on Eq. (1), as the magnitude of the DEP force will increase with the droplet size, a higher electric voltage should be applied for the separation of oil droplets with smaller diameters such as $7.5 \mu \mathrm{m}$ and $11 \mu \mathrm{m}$. Figure 4(B) shows that the mixture of the oil droplets with two different sizes, $7.5 \mu \mathrm{m}$ and $11 \mu \mathrm{m}$, were successfully separated under $\phi_{C}=320 \mathrm{~V}$. It should be noted that this nano-orifice based DC-DEP separation system can be employed for continuously sorting smaller oil droplets of $7.5 \mu \mathrm{m}$ and $11 \mu \mathrm{m}$ with a high separation resolution of $3.5 \mu \mathrm{m}$ (difference in diameter) as illustrated in Figure 4(B). In addition, by controlling the applied voltages to electrodes $C$, the separation of oil droplets of different sizes can be achieved. Thus, the applied voltages can be optimized for the specific size of the targeted droplets by theoretical predictions and experimental calibrations.

\subsection{Separation of oil droplets by content}

In this work, in order to examine the sensitivity of this nano-orifice based DC-DEP separation system, the separation of the oil droplets of similar sizes but different contents, i.e., the droplets of silicone oil and carbon grease with a diameter of $7.5 \mu \mathrm{m}$, the droplets of silicone oil and carbonplus-silver grease with a diameter of $7 \mu \mathrm{m}$, as well as the droplets of carbon grease and carbonplus-silver grease with a diameter of $7.5 \mu \mathrm{m}$ were conducted under $\phi_{C}=320 \mathrm{~V}, \phi_{C}=360 \mathrm{~V}$, and $\phi_{C}=360 \mathrm{~V}$, respectively. The physicochemical properties of these oil droplets are listed in Table 1. As shown in Figure 5, the $f_{\mathrm{CM}}$ values of the oil droplets with three different contents are plotted varying with the conductivity of the surrounding solution, $\sigma_{m}$. In order to make sure that the oil droplets with different contents experience the p-DEP and n-DEP forces, respectively, the suspending solutions with two different electrical conductivities were used. The solution of 0.4 $\mathrm{mM} \mathrm{K}_{2} \mathrm{HPO}_{4}$ with a pH 7 and a conductivity of $1 \times 10^{-2} \mathrm{~S} / \mathrm{m}$ was used for the sorting of the 
droplets of carbon grease and carbon-plus-silver grease, and the DI water with $\mathrm{pH}$ of 7 and a conductivity of $5.5 \times 10^{-6} \mathrm{~S} / \mathrm{m}$ for the rest groups of separation.

The separation of the oil droplets of similar sizes but different contents was conducted. By selecting the surrounding solution with a specific electrical conductivity, the separation of the droplets of silicone oil and carbon grease with a diameter of $7.5 \mu \mathrm{m}$, the droplets of silicone oil and carbon-plus-silver grease with a diameter of $7 \mu \mathrm{m}$, and the droplets of carbon grease and carbon-plus-silver grease with a diameter of $7.5 \mu \mathrm{m}$ based on the opposite dielectrophoretic behaviors as a function of the droplet content under the condition of $\phi_{C}=320 \mathrm{~V}, \phi_{C}=360 \mathrm{~V}$, as well as $\phi_{C}=360 \mathrm{~V}$, respectively, is shown in Figure 6 .

In Figure 6(A), as the electrical conductivity of the droplets of carbon grease is higher than that of the surrounding solution, i.e., $f_{\mathrm{CM}} \approx 1$ (Table 1 ), these droplets were attracted by the $\mathrm{p}$-DEP forces towards the electric field maximum area and flowed into the outlet branch E. On the contary, the droplets of silicone oil have an electrical conductivity smaller than that of the medium, i.e., $f_{\mathrm{CM}} \approx$ -0.5 (Table 1), the droplets experienced n-DEP effects and were repelled from electric field maximum region and flowed into the outlet branch F. It clearly shows in Figure 6(B) and 6(C) that the mixtures of the droplets of carbon-plus-silver grease $\left(f_{\mathrm{CM}} \approx 1\right)$ and silicon oil $\left(f_{\mathrm{CM}} \approx-0.5\right)$ with a diameter of $7 \mu \mathrm{m}$, and the droplets of carbon grease $\left(f_{\mathrm{CM}} \approx 1\right)$ and carbon-plus-silver grease $\left(f_{\mathrm{CM}} \approx-0.43\right)$ with a diameter of $7.5 \mu \mathrm{m}$ were successfully separated by experiencing $\mathrm{p}$-DEP and n-DEP forces, respectively. This indicates that the continuous separation of the oil droplets of similar sizes but different contents based on these opposite DEP behaviors can be achieved in this nano-orifice based DC-DEP separation device. Furthermore, it should be noticed that the separation of the oil droplets of similar sizes with other different contents can be achieved by simply controlling the applied voltages and selecting the suspending solution with a specific electrical conductivity. The relationships among the voltages, electrical conductivity, and the content of the oil droplets can be easily obtained through numerical simulation and experiments.

\section{Conclusion}

The nano-orifice based DC-DEP method is developed to separate the oil droplets by size and by content under a pressure-driven flow in the nanofluidic chip for the first time. The size-dependent separation of smaller silicone oil droplets with a small size difference of only $3.5 \mu \mathrm{m}$ was 
demonstrated. Furthermore, by selecting the surrounding solution with a specific electrical conductivity, the separation of the oil droplets of similar size but different contents can be achieve by opposite DEP effects, i.e., p-DEP and n-DEP, respectively in the nano-orifice based DC-DEP device. In this study, by applying a low DC electric potential difference through a pair of the nano and micron orifices with a large width ratio on the opposite sidewall, a stronger non-uniformity of the electric field gradient is generated and hence sufficient greater DEP effects are achieved, leading to a high separation resolution, whereas the complicated implementation of microelectrodes inside the microfluidic chips [44,45,48,56-58] and expensive fabrication processes are avoided. Moreover, in this system, the production of non-uniformity of the electric field does not change the cross-section of the microfluidic channel and has no influence on liquid flow and the droplets transportation. By using the pressure-driven flow to drive the droplets, the throughput can be dramatically increased in comparison with other microfluidic DEP methods $[39,40,43]$. In addition, by simply adjusting the applied voltage and the electrical conductivity of the surrounding solution, the separation of target oil droplets with specific size and content can be achieved. This paper, for the first time to our knowledge, presents a simple and effective method using DC-DEP to separate oil droplets by size and by content for wide applications in industry and research. Further study is under way to separate different kinds of oil droplets carrying different biomolecules or bioparticles such as nucleic acid or bacterial.

\section{Acknowledgements}

The authors wish to thank the Natural Sciences and Engineering Research Council (NSERC) of Canada for financial support through a research grant to Dr. Li.

\section{References}

[1] J.R. Millman, K.H. Bhatt, B.G. Prevo, O.D. Velev, Anisotropic particle synthesis in dielectrophoretically controlled microdroplet reactors., Nat. Mater. 4 (2005) 98-102. doi:10.1038/nmat1270.

[2] B.G. De Geest, J.P. Urbanski, T. Thorsen, J. Demeester, S.C. De Smedt, Synthesis of monodisperse biodegradable microgels in microfluidic devices, Langmuir. 21 (2005) 10275-10279. doi:10.1021/la051527y. 
[3] W.W. Yang, Y.C. Lu, Z.Y. Xiang, G.S. Luo, Monodispersed microcapsules enclosing ionic liquid of 1-butyl-3-methylimidazolium hexafluorophosphate, React. Funct. Polym. 67 (2007) 81-86. doi:10.1016/j.reactfunctpolym.2006.10.005.

[4] S. Sakai, I. Hashimoto, K. Kawakami, Usefulness of flow focusing technology for producing subsieve-size cell enclosing capsules: Application for agarose capsules production, Biochem. Eng. J. 30 (2006) 218-221. doi:10.1016/j.bej.2006.04.003.

[5] A. Kato, E. Shindo, T. Sakaue, A. Tsuji, K. Yoshikawa, Conformational transition of giant DNA in a confined space surrounded by a phospholipid membrane, Biophys. J. 97 (2009) 1678-1686. doi:10.1016/j.bpj.2009.06.041.

[6] J. Clausell-Tormos, D. Lieber, J.C. Baret, A. El-Harrak, O.J. Miller, L. Frenz, J. Blouwolff, K.J. Humphry, S. Köster, H. Duan, C. Holtze, D.A. Weitz, A.D. Griffiths, C.A. Merten, Droplet-Based Microfluidic Platforms for the Encapsulation and Screening of Mammalian Cells and Multicellular Organisms, Chem. Biol. 15 (2008) 427-437. doi:10.1016/j.chembiol.2008.04.004.

[7] S. Akbari, T. Pirbodaghi, A droplet-based heterogeneous immunoassay for screening single cells secreting antigen-specific antibodies., Lab Chip. 14 (2014) 3275-80. doi:10.1039/c4lc00082j.

[8] W.W. Chen, L. Balaj, L.M. Liau, M.L. Samuels, S.K. Kotsopoulos, C.A. Maguire, L. Loguidice, H. Soto, M. Garrett, L.D. Zhu, S. Sivaraman, C. Chen, E.T. Wong, B.S. Carter, F.H. Hochberg, X.O. Breakefield, J. Skog, BEAMing and Droplet Digital PCR Analysis of Mutant IDH1 mRNA in Glioma Patient Serum and Cerebrospinal Fluid Extracellular Vesicles., Mol. Ther. Nucleic Acids. 2 (2013) e109. doi:10.1038/mtna.2013.28.

[9] G.S. Du, J.Z. Pan, S.P. Zhao, Y. Zhu, J.M.J. Den Toonder, Q. Fang, Cell-based drug combination screening with a microfluidic droplet array system, Anal. Chem. 85 (2013) 6740-6747. doi:10.1021/ac400688f.

[10] H.N. Joensson, H. Andersson Svahn, Droplet microfluidics-A tool for single-cell analysis, Angew. Chemie - Int. Ed. 51 (2012) 12176-12192. doi:10.1002/anie.201200460.

[11] M.T. Guo, A. Rotem, J.A. Heyman, D.A. Weitz, Droplet microfluidics for high-throughput biological assays, Lab Chip. 12 (2012) 2146. doi:10.1039/c2lc21147e.

[12] H. Song, R.F. Ismagilov, Millisecond Kinetics on a Microfluidic Chip Using Nanoliters of Reagents, J. Am. Chem. Soc. 125 (2003) 14613-14619. doi:10.1021/ja0354566. 
[13] T. Henkel, T. Bermig, M. Kielpinski, A. Grodrian, J. Metze, J.M. Köhler, Chip modules for generation and manipulation of fluid segments for micro serial flow processes, Chem. Eng. J. 101 (2004) 439-445. doi:10.1016/j.cej.2004.01.021.

[14] J. Ju, C. Zeng, L. Zhang, N. Xu, Continuous synthesis of zeolite NaA in a microchannel reactor, Chem. Eng. J. 116 (2006) 115-121. doi:10.1016/j.cej.2005.11.006.

[15] R. Zilionis, J. Nainys, A. Veres, V. Savova, D. Zemmour, A.M. Klein, L. Mazutis, Singlecell barcoding and sequencing using droplet microfluidics., Nat. Protoc. 12 (2017) 44-73. doi:10.1038/nprot.2016.154.

[16] S.-Y. Teh, R. Lin, L.-H. Hung, A.P. Lee, Droplet microfluidics., Lab Chip. 8 (2008) 198220. doi:10.1039/b715524g.

[17] L.L. Schramm, Emulsions, Foams, and Suspensions: Fundamentals and Applications, 2006. doi:10.1002/3527606750.

[18] D. Ganguli, M. Ganguli, Inorganic Particle Synthesis via Macro and Microemulsions: A Micrometer to Nanometer Landscape, Springer Science \& Business Media, 2003. doi:10.1007/978-1-4615-0047-6.

[19] T. Nisisako, T. Torii, T. Takahashi, Y. Takizawa, Synthesis of monodisperse bicolored janus particles with electrical anisotropy using a microfluidic co-flow system, Adv. Mater. 18 (2006) 1152-1156. doi:10.1002/adma.200502431.

[20] J.H. Xu, S.W. Li, J. Tan, Y.J. Wang, G.S. Luo, Preparation of highly monodisperse droplet in a T-junction microfluidic device, AIChE J. 52 (2006) 3005-3010. doi:10.1002/aic.

[21] T. Nisisako, T. Torii, T. Higuchi, Droplet formation in a microchannel network., Lab Chip. 2 (2002) 24-26. doi:10.1039/b108740c.

[22] C. Priest, S. Herminghaus, R. Seemann, Generation of monodisperse gel emulsions in a microfluidic device, Appl. Phys. Lett. 88 (2006) 1-3. doi:10.1063/1.2164393.

[23] T. Thorsen, R.W. Roberts, F.H. Arnold, S.R. Quake, Dynamic pattern formation in a vesiclegenerating microfluidic device, Phys. Rev. Lett. 86 (2001) 4163-4166. doi:10.1103/PhysRevLett.86.4163.

[24] S. Takeuchi, P. Garstecki, D.B. Weibel, G.M. Whitesides, An axisymmetric flow-focusing microfluidic device, Adv. Mater. 17 (2005) 1067-1072. doi:10.1002/adma.200401738.

[25] A. Woodward, T. Cosgrove, J. Espidel, P. Jenkins, N. Shaw, Monodisperse emulsions from a microfluidic device, characterised by diffusion NMR, Soft Matter. 3 (2007) 627-633. 
doi:10.1039/b616463n.

[26] L. Yobas, S. Martens, W.-L. Ong, N. Ranganathan, High-performance flow-focusing geometry for spontaneous generation of monodispersed droplets., Lab Chip. 6 (2006) 10731079. doi:10.1039/b602240e.

[27] S.L. Anna, N. Bontoux, H.A. Stone, Formation of dispersions using "flow focusing" in microchannels, Appl. Phys. Lett. 82 (2003) 364-366. doi:10.1063/1.1537519.

[28] R. Ahmed, T.B. Jones, Dispensing picoliter droplets on substrates using dielectrophoresis, J. Electrostat. 64 (2006) 543-549. doi:10.1016/j.elstat.2005.10.008.

[29] T.B. Jones, Liquid dielectrophoresis on the microscale, J. Electrostat. 51-52 (2001) 290299. doi:10.1016/S0304-3886(01)00074-2.

[30] T.B. Jones, M. Gunji, M. Washizu, M.J. Feldman, Dielectrophoretic liquid actuation and nanodroplet formation, J. Appl. Phys. 89 (2001) 1441-1448. doi:10.1063/1.1332799.

[31] S.K. Cho, H. Moon, C.J. Kim, Creating, transporting, cutting, and merging liquid droplets by electrowetting-based actuation for digital microfluidic circuits, J. Microelectromechanical Syst. 12 (2003) 70-80. doi:10.1109/JMEMS.2002.807467.

[32] J.M. Roux, Y. Fouillet, J.L. Achard, 3D droplet displacement in microfluidic systems by electrostatic actuation, Sensors Actuators, A Phys. 134 (2007) 486-493. doi:10.1016/j.sna.2006.05.012.

[33] J. Berthier, P. Clementz, O. Raccurt, D. Jary, P. Claustre, C. Peponnet, Y. Fouillet, Computer aided design of an EWOD microdevice, Sensors Actuators, A Phys. 127 (2006) 283-294. doi:10.1016/j.sna.2005.09.026.

[34] G.F. Christopher, S.L. Anna, Microfluidic methods for generating continuous droplet streams, J. Phys. D. Appl. Phys. 40 (2007) R319-R336. doi:10.1088/0022-3727/40/19/R01.

[35] C.T. Chen, G.B. Lee, Formation of microdroplets in liquids utilizing active pneumatic choppers on a microfluidic chip, J. Microelectromechanical Syst. 15 (2006) 1492-1498. doi:10.1109/JMEMS.2006.883572.

[36] Y.-C. Tan, A.P. Lee, Microfluidic separation of satellite droplets as the basis of a monodispersed micron and submicron emulsification system., Lab Chip. 5 (2005) 1178-83. doi:10.1039/b504497a.

[37] Y.-C. Tan, J.S. Fisher, A.I. Lee, V. Cristini, A.P. Lee, Design of microfluidic channel geometries for the control of droplet volume, chemical concentration, and sorting., Lab Chip. 
4 (2004) 292-298. doi:10.1039/b403280m.

[38] Y.C. Tan, Y.L. Ho, A.P. Lee, Droplet coalescence by geometrically mediated flow in microfluidic channels, Microfluid. Nanofluidics. 3 (2007) 495-499. doi:10.1007/s10404006-0136-1.

[39] D. Huh, J.H. Bahng, Y. Ling, H.H. Wei, O.D. Kripfgans, J.B. Fowlkes, J.B. Grotberg, S. Takayama, Gravity-driven microfluidic particle sorting device with hydrodynamic separation amplification, Anal. Chem. 79 (2007) 1369-1376. doi:10.1021/ac061542n.

[40] A.R. Abate, J.J. Agresti, D.A. Weitz, Microfluidic sorting with high-speed single-layer membrane valves, Appl. Phys. Lett. 96 (2010). doi:10.1063/1.3431281.

[41] C.W. Lai, Y.H. Lin, G. Bin Lee, A microfluidic chip for formation and collection of emulsion droplets utilizing active pneumatic micro-choppers and micro-switches, Biomed. Microdevices. 10 (2008) 749-756. doi:10.1007/s10544-008-9186-3.

[42] J. Kr\$uuml\$ger, K. Singh, A. O\$apos\$Neill, C. Jackson, A. Morrison, P. O\$apos\$Brien, Development of a microfluidic device for fluorescence activated cell sorting, J. Micromechanics Microengineering. 12 (2002) 486-494. doi:10.1088/0960-1317/12/4/324.

[43] S.K. Cho, Y. Zhao, C.-J.C. Kim, Concentration and binary separation of micro particles for droplet-based digital microfluidics., Lab Chip. 7 (2007) 490-8. doi:10.1039/b615665g.

[44] K. Ahn, C. Kerbage, T.P. Hunt, R.M. Westervelt, D.R. Link, D.A. Weitz, Dielectrophoretic manipulation of drops for high-speed microfluidic sorting devices, Appl. Phys. Lett. 88 (2006) 1-3. doi:10.1063/1.2164911.

[45] S.H. Hung, Y.H. Lin, G. Bin Lee, A microfluidic platform for manipulation and separation of oil-in-water emulsion droplets using optically induced dielectrophoresis, J. Micromechanics Microengineering. 20 (2010) 45026. doi:10.1088/0960-1317/20/4/045026.

[46] J.-C. Baret, O.J. Miller, V. Taly, M. Ryckelynck, A. El-Harrak, L. Frenz, C. Rick, M.L. Samuels, J.B. Hutchison, J.J. Agresti, D.R. Link, D.A. Weitz, A.D. Griffiths, Fluorescenceactivated droplet sorting (FADS): efficient microfluidic cell sorting based on enzymatic activity., Lab Chip. 9 (2009) 1850-8. doi:10.1039/b902504a.

[47] T.P. Hunt, D. Issadore, R.M. Westervelt, Integrated circuit/microfluidic chip to programmably trap and move cells and droplets with dielectrophoresis., Lab Chip. 8 (2008) 81-87. doi:10.1039/b710928h.

[48] F. Guo, X. Ji, K. Liu, R. He, L. Zhao, Z. Guo, W. Liu, S. Guo, X. Zhao, Droplet electric 
separator microfluidic device for cell sorting, Appl. Phys. Lett. 96 (2010) 193701. doi:10.1063/1.2164911.

[49] K. Zhao, D. Li, Continuous separation of nanoparticles by type via localized DCDielectrophoresis using asymmetric nano-orifice in pressure-driven flow, Sensors Actuators B Chem. 250 (2017) 274-284. doi:10.1016/j.snb.2017.04.184.

[50] K. Zhao, D. Li, Numerical studies of manipulation and separation of Janus particles in nanoorifice based DC- Dielectrophoretic microfluidic chips, J. Micromechanics Microengineering. (2017). doi:10.1088/1361-6439/aa7eae.

[51] K. Zhao, R. Peng, D. Li, Separation of nanoparticles by a nano-orifice based DCDielectrophoresis method in pressure-driven flow, Nanoscale. 8 (2016) 18945-18955. doi:10.1039/C6NR06952E.

[52] L.M. Fidalgo, G. Whyte, D. Bratton, C.F. Kaminski, C. Abell, W.T.S. Huck, From microdroplets to microfluidics: Selective emulsion separation in microfluidic devices, Angew. Chemie - Int. Ed. 47 (2008) 2042-2045. doi:10.1002/anie.200704903.

[53] S.I. Han, H. Soo Kim, A. Han, In-droplet cell concentration using dielectrophoresis, Biosens. Bioelectron. 97 (2017) 41-45. doi:10.1016/j.bios.2017.05.036.

[54] Y. Kang, B. Cetin, Z. Wu, D. Li, Continuous particle separation with localized ACdielectrophoresis using embedded electrodes and an insulating hurdle, Electrochim. Acta. 54 (2009) 1715-1720. doi:10.1016/j.electacta.2008.09.062.

[55] B. Çetin, D. Li, Dielectrophoresis in microfluidics technology, Electrophoresis. 32 (2011) 2410-2427. doi:10.1002/elps.201100167.

[56] S.-Y. Park, S. Kalim, C. Callahan, M.A. Teitell, E.P.Y. Chiou, A light-induced dielectrophoretic droplet manipulation platform., Lab Chip. 9 (2009) 3228-3235. doi:10.1039/b909158k.

[57] D.H. Lee, H. Hwang, J.K. Park, Generation and manipulation of droplets in an optoelectrofluidic device integrated with microfluidic channels, Appl. Phys. Lett. 95 (2009). doi:10.1063/1.3253411.

[58] J.-P. Delville, M.R.D. Saint Vincent, R.D. Schroll, H. Chraibi, B. Issenmann, R. Wunenburger, D. Lasseux, W.W. Zhang, E. Brasselet, Laser microfluidics: fluid actuation by light, J. Opt. A Pure Appl. Opt. 11 (2009) 34015. doi:10.1088/1464-4258/11/3/034015.

[59] T B Jones, Electromechanics of particles, Cambridge University Press, 1995. 
doi:10.1016/S0032-5910(97)82724-6.

[60] G.H. Markx, P.A. Dyda, R. Pethig, Dielectrophoretic separation of bacteria using a conductivity gradient, J. Biotechnol. 51 (1996) 175-180. doi:10.1016/01681656(96)01617-3.

[61] R. Peng, D. Li, Fabrication of nanochannels on polystyrene surface, Biomicrofluidics. 9 (2015) 24117. doi:10.1063/1.4918643.

[62] K.M. Choi, J.A. Rogers, A photocurable poly(dimethylsiloxane) chemistry designed for soft lithographic molding and printing in the nanometer regime, J. Am. Chem. Soc. 125 (2003) 4060-4061. doi:10.1021/ja029973k. 


\section{List of figures}

Figure 1. (A) Schematic diagram of the microfluidic DC-DEP chip with a pair of asymmetric orifices for droplets separation. (B) Schematic diagram of the experimental system.

Figure 2. (A) An example of the nano-orifice based device for the droplets separation. (B) A zoomin photograph of the asymmetric orifices area captured by using the microscope. (C) Illustration of a 3D view of a PDMS nanochannel section measured by AFM. (D) A cross-section of the nanochannel with a size about $860 \pm 19 \mathrm{~nm}$ in width and $290 \pm 22 \mathrm{~nm}$ in depth.

Figure 3. The trajectory of the oil droplets and distribution of the electric field in the nanofluidic channel. (A) An example of the separation of the $7.5 \mu \mathrm{m}$ and $11 \mu \mathrm{m}$ oil droplets in the channel. (B) Distribution of the gradient of the electric field squared $\left(\nabla|E|^{2}\right)$. The width and length of the small orifice is $860 \mathrm{~nm}$ and $15 \mu \mathrm{m}$, respectively, and the width of the large orifice is $125 \mu \mathrm{m}$. The voltage applied is $320 \mathrm{~V}$ in electrode $\mathrm{C}$ and zero in electrode $\mathrm{D}$.

Figure 4. Separation of the oil droplets by size. (A) Separation of $9 \mu \mathrm{m}$ and $14.5 \mu \mathrm{m}$ diameter silicone oil droplets, $\phi_{C}=240 \mathrm{~V}, \phi_{D}=0 \mathrm{~V}$. (B) separation of $7.5 \mu \mathrm{m}$ and $11 \mu \mathrm{m}$ diameter silicone oil droplets, $\phi_{C}=320 \mathrm{~V}, \phi_{D}=0 \mathrm{~V}$. $\phi_{C}$ and $\phi_{D}$ indicate the voltages applied to the reserviors $\mathrm{C}$ and D. In this chip, the width and length of the small orifice is $860 \mathrm{~nm}$ and $15 \mu \mathrm{m}$, respectively, and the width of the large orifice is $125 \mu \mathrm{m}$.

Figure 5. Clausius-Mossotti factor $f_{\mathrm{CM}}$ for the oil droplets with three different contents as a function of the electric conductivity of the suspending solution $\sigma_{m}$.

Figure 6. Separation of the oil droplets of similar sizes by content. (A) Separation of the silicon oil droplets and carbon grease droplets with a diameter of $7.5 \mu \mathrm{m}, \phi_{C}=320 \mathrm{~V}, \phi_{D}=0 \mathrm{~V}$. (B) Separation of the silicon oil droplets and carbon-plus-silver grease droplets with a diameter of 7 $\mu \mathrm{m}, \phi_{C}=360 \mathrm{~V}, \phi_{D}=0 \mathrm{~V}$. (C) Separation of the carbon grease droplets and carbon-plus-silver grease droplets with a diameter of $7.5 \mu \mathrm{m}, \phi_{C}=360 \mathrm{~V}, \phi_{D}=0 \mathrm{~V}$. The width and length of the small orifice is $860 \mathrm{~nm}$ and $15 \mu \mathrm{m}$, respectively, and the width of the large orifice is $125 \mu \mathrm{m}$. The small dots are the impurities of grease. 


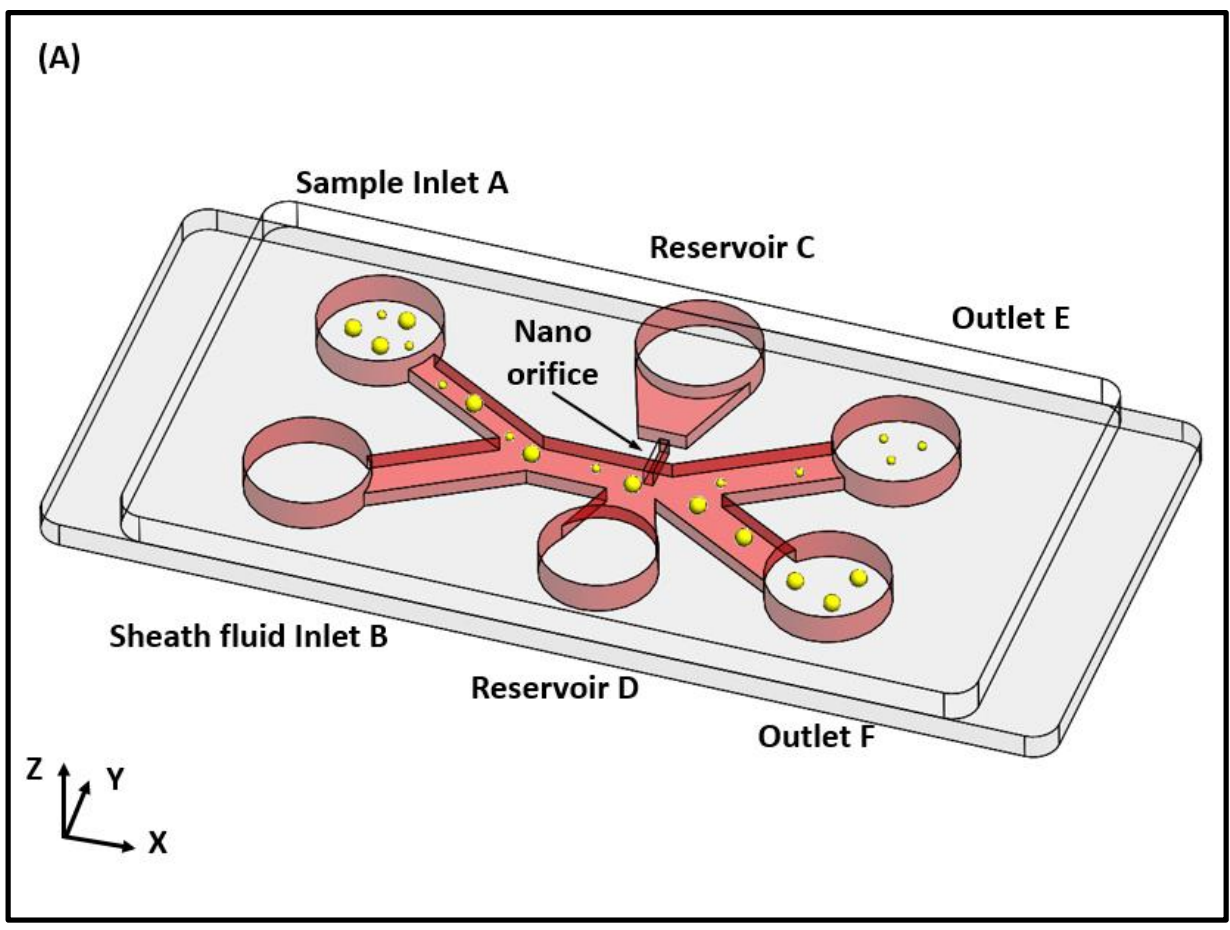

(B)

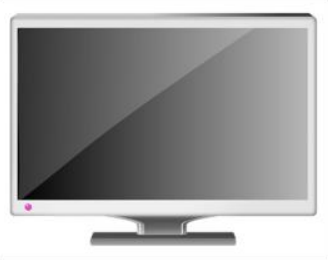

Computer

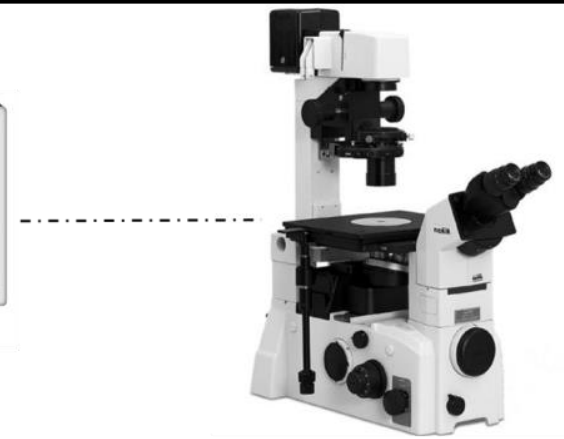

Microscope

DC Power Supply

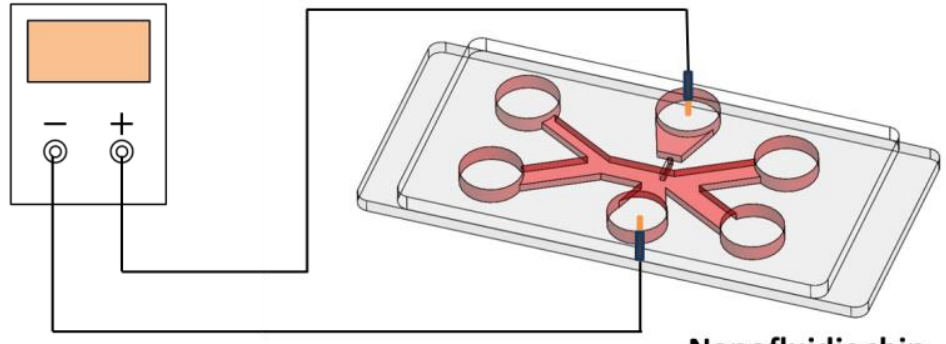

Nanofluidic chip

Figure 1 

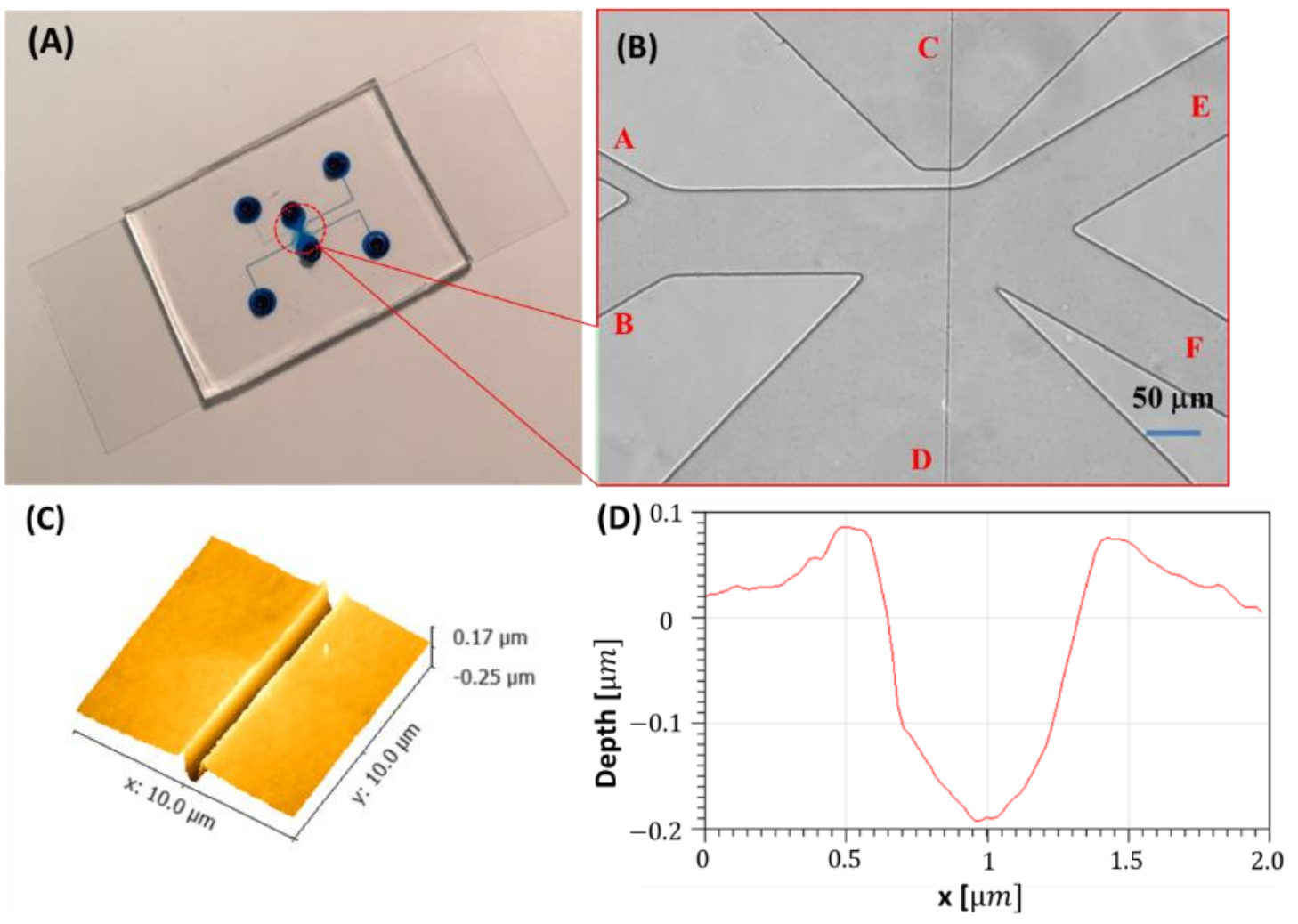

Figure 2 

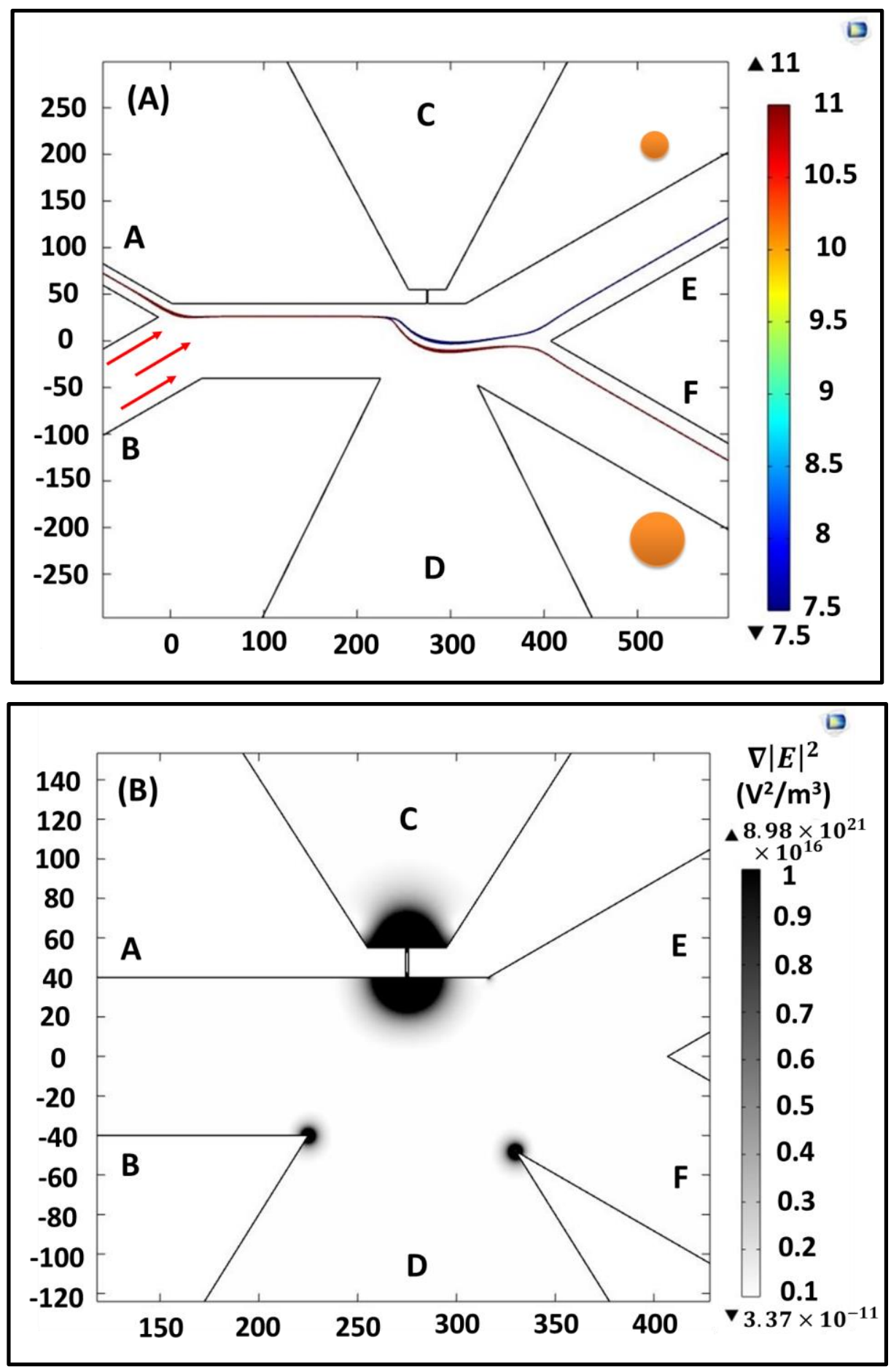

Figure 3 


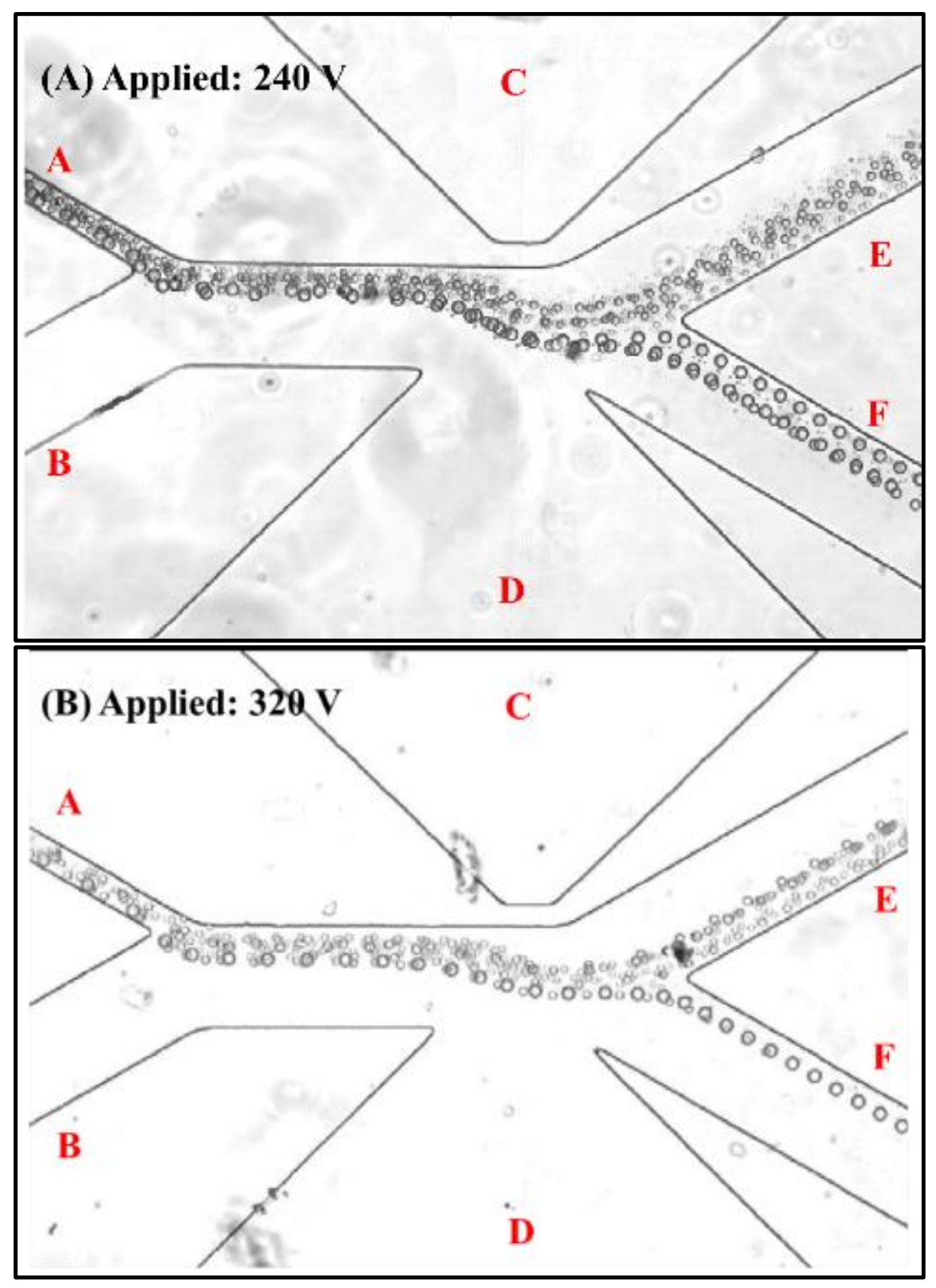

Figure 4 


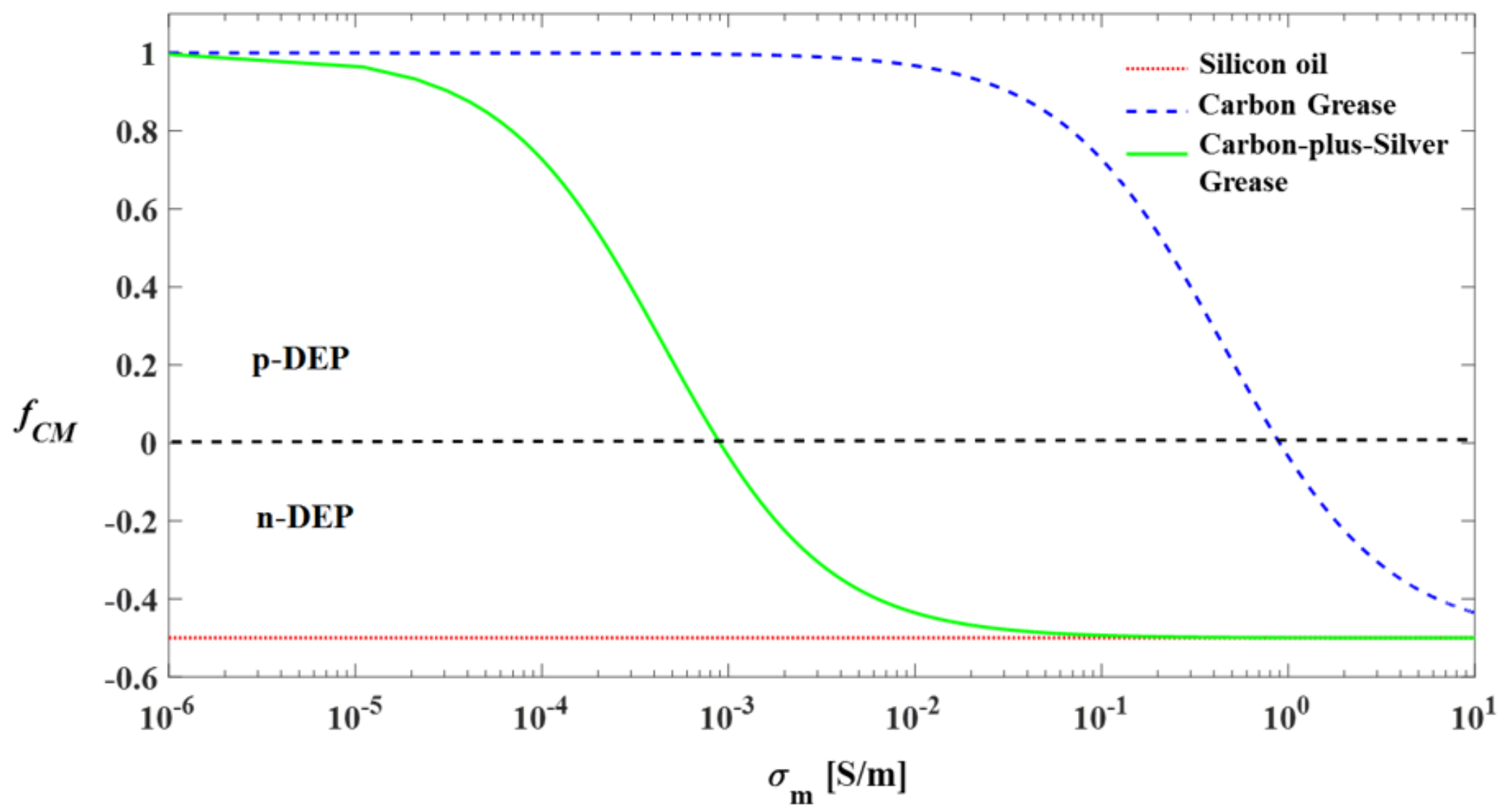

Figure 5 


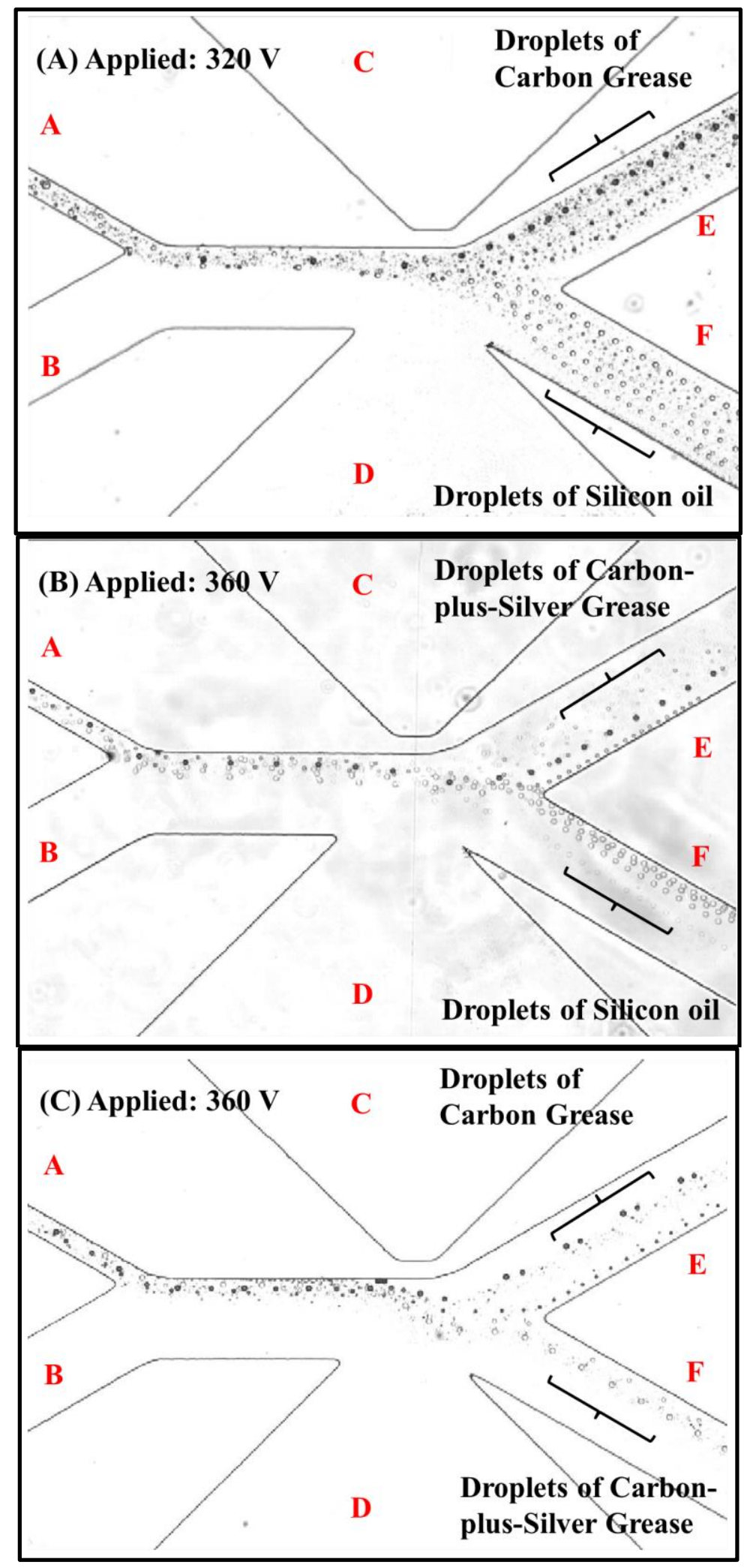

Figure 6 
Table 1. Physicochemical properties of three oil droplets and the corresponding Clausius-Mossotti factor $\left(f_{\mathrm{CM}}\right)$ for the oil droplets in the suspending medium with two different electric conductivities, i.e., $\sigma_{\mathrm{m}}=0.01 \mathrm{~S} / \mathrm{m}$ for the droplets of carbon grease and carbon-plus-silver grease, and $\sigma_{\mathrm{m}}=5.5$ $\times 10^{-6} \mathrm{~S} / \mathrm{m}$ for the rest two groups.

\begin{tabular}{|c|c|c|c|}
\hline Property & $\begin{array}{l}\text { Conductivity } \\
\qquad(\mathrm{S} / \mathrm{m})\end{array}$ & $\begin{array}{l}f_{\mathrm{CM}} \text { with } \sigma_{\mathrm{m}}= \\
5.5 \times 10^{-6} \mathrm{~S} / \mathrm{m}\end{array}$ & $\begin{array}{l}f_{\mathrm{CM}} \text { with } \sigma_{m}= \\
1 \times 10^{-2} \mathrm{~S} / \mathrm{m}\end{array}$ \\
\hline $\begin{array}{l}\text { Droplet of Silicon oil } \\
\text { (Transparent in color) }\end{array}$ & $10^{-13}$ & $\approx-0.5$ & \\
\hline Droplet of Carbon grease & & & \\
\hline $\begin{array}{l}\text { (Black in color. Silicon oil based, } \\
\text { conductive filler: carbon.) }\end{array}$ & 0.9 & $\approx 1$ & $\approx 1$ \\
\hline Droplet of Carbon-plus-Silver grease & & & \\
\hline $\begin{array}{l}\text { (Silver in color. Silicon oil based, } \\
\text { conductive filler: carbon-plus-silver) }\end{array}$ & $0.9 \times 10^{-3}$ & $\approx 1$ & $\approx-0.43$ \\
\hline
\end{tabular}

